\title{
Optodynamic Phenomena During Laser-Activated Irrigation Within Root Canals
}

\author{
Nejc Lukač ${ }^{1}$ - Peter Gregorčič ${ }^{1}$ • Matija Jezeršek ${ }^{1}$
}

Received: 10 October 2015 / Accepted: 28 April 2016/ Published online: 10 May 2016 (C) The Author(s) 2016. This article is published with open access at Springerlink.com

\begin{abstract}
Laser-activated irrigation is a powerful endodontic treatment for smear layer, bacteria, and debris removal from the root canal. In this study, we use shadow photography and the laser-beam-transmission probe to examine the dynamics of laserinduced vapor bubbles inside a root canal model and compare ultrasonic needle irrigation to the laser method. Results confirm important phenomenological differences in the two endodontic methods with the laser method resulting in much deeper irrigation. Observations of simulated debris particles show liquid vorticity effects which in our opinion represents the major cleaning mechanism.
\end{abstract}

Keywords Er:YAG · Laser-beam-transmission probe $\cdot$ Root canal ·

Shadow photography · Ultrasonic irrigation · Vapor bubble

\section{Introduction}

Laser-activated irrigation is a powerful endodontic treatment for smear layer, bacteria, and debris removal from the root canal $[1,2]$. Recent studies have shown that Er:YAG laser irrigation has much higher efficacy [1-5] compared to traditional methods such as syringe or ultrasonic needle irrigation. This is attributed to optodynamic phenomena during Er:YAG laser pulse delivery. Pulses are delivered into the canal using a fiber tip. High absorption of this light in water (wavelength $2.94 \mu \mathrm{m}$; low

This article is part of the selected papers presented at the 18th International Conference on Photoacoustic and Photothermal Phenomena.

$\bowtie \quad$ Matija Jezeršek

Matija.jezersek@fs.uni-lj.si

1 Faculty of Mechanical Engineering, University of Ljubljana, Aškerčeva 6, 1000 Ljubljana, Slovenia 
light penetration depth) leads to explosive boiling that generates vapor bubbles [6,7], causing mixing of liquid also at distant regions of the complex root canal anatomy. This is an important advantage over ultrasonic needle irrigation, where a significant effect occurs only in the close proximity of the instrument [8]. Furthermore, ultrasonic irrigation is problematic in curved and complex root canal geometries as efficiency is reduced by the instrument touching the canal walls [9]. Laser-induced cavitation in water has been studied thoroughly $[10,11]$; however, these results are not always directly applicable to phenomena inside the confined volume of a root canal and the mechanisms of laser-activated irrigation of root canals is still not completely understood.

The aim of this study is to better understand the above-mentioned phenomena. For this purpose, an experimental setup was developed based on two measuring principles. Shadow photography with short, $10 \mathrm{~ns}$, white-light illumination flashes, and frequencydoubled Nd:YAG laser illumination is used to visualize the cavitation bubble dynamics [7] inside the root canal. Simulated debris particles within the transparent model of the root canal are used to examine liquid vorticity. A laser-beam-transmission probe (LBTP) is simultaneously used to measure the oscillation of the cavitation bubble and confirm the results of the water vorticity observations. The laser endodontic method is firstly compared to ultrasonic root canal irrigation.

\section{Experimental Setup}

\subsection{Vapor Bubble Dynamics and Ultrasound Irrigation Experimental Setup}

The experimental setup is shown in Fig.1a. The root canal model is composed of a plastic cutout clamped between two transparent plates and submerged in a glass container filled with distilled water. A syringe is used to break the surface tension preventing the air from escaping the root canal model, and the additional water is pumped into the model to ensure it is completely filled with liquid. A suspension of optical polishing powder is used in the canal to simulate debris. A conical fiber tip (Fotona d.d., Slovenia, Xpulse, 400/14) is positioned inside the root canal model $3 \mathrm{~mm}$ below the upper edge of the cutout. An Er:YAG dental laser $(\lambda=2940 \mathrm{~nm}$, Fotona d.d., Slovenia, Lightwalker) is used to deliver $50 \mathrm{~mJ}, 100 \mu \mathrm{s}$ long pulses into the root canal with a frequency of $15 \mathrm{~Hz}$. The excitation laser, camera, and illumination flash are synchronized by a microcontroller controlled by a PC with custom-developed software written in Labview. White-light, $10 \mathrm{~ns}$ long illumination flash are generated by a nanosecond flash lamp (HSPS, Germany, NanoLite KL-K). Images of the vapor bubble are captured with a digital single lens reflex camera (Nikon, Japan, D90). An image is captured after each laser pulse with varying delays between the illumination and excitation pulses, producing a sequence of the bubble's oscillation. The method relies on the repeatability of the studied phenomenon. 


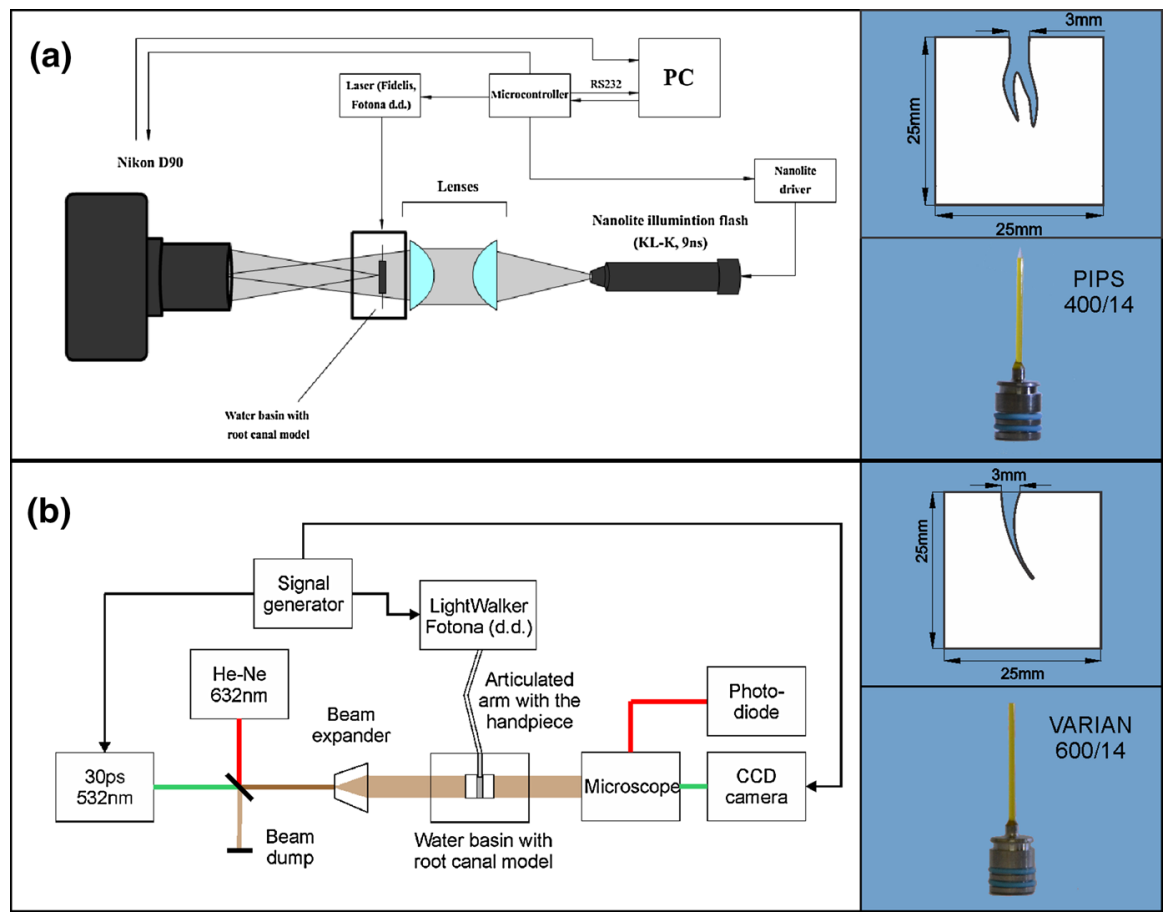

Fig. 1 Experimental setup for (a) shadow photography and (b) water vorticity experiments. The different root canal geometries and FTs used in the experiments are shown in the insets on the right of the experimental setup

\subsection{Water Vorticity Measuring Setup}

In water vorticity experiments, the dynamics inside the root canal is observed with a LBTP and the system of shadow photography is described in Fig. 1b (see also Ref. [10]). For the LBTP, a He-Ne laser $(\lambda=633 \mathrm{~nm})$ guided through a beam expander is used to illuminate the area of interest and a photodiode (Thorlabs, USA, PDA10AEC) is used to measure transmitted light power. The illumination source for shadow photography is a frequency-doubled Nd:YAG laser $(\lambda=532 \mathrm{~nm}$, Ekspla, Lithuania, PL2250_SH_TH) with pulse duration of $30 \mathrm{ps}$. The images are acquired with a CCD camera (Basler AG, Germany, scA1400-17fm, 1.4Mpx). A flat fiber tip (Fotona d.d., Slovenia, VARIAN, 600/14) is positioned $3 \mathrm{~mm}$ below the upper edge of the root canal. In this experiment, the root canal model was partially filled with flint particles roughly $100 \mu \mathrm{m}$ in diameter to simulate debris. A series of laser pulses with durations of $100 \mu \mathrm{s}$, energies of $30 \mathrm{~mJ}$, and frequency of $0.1 \mathrm{~Hz}$ were delivered through the fiber tip positioned inside the root canal model. The low frequency was chosen to prevent cross-talk between subsequent laser pulses and the energy of the laser pulses was lowered so that the cavitation bubbles did not directly touch the debris. 


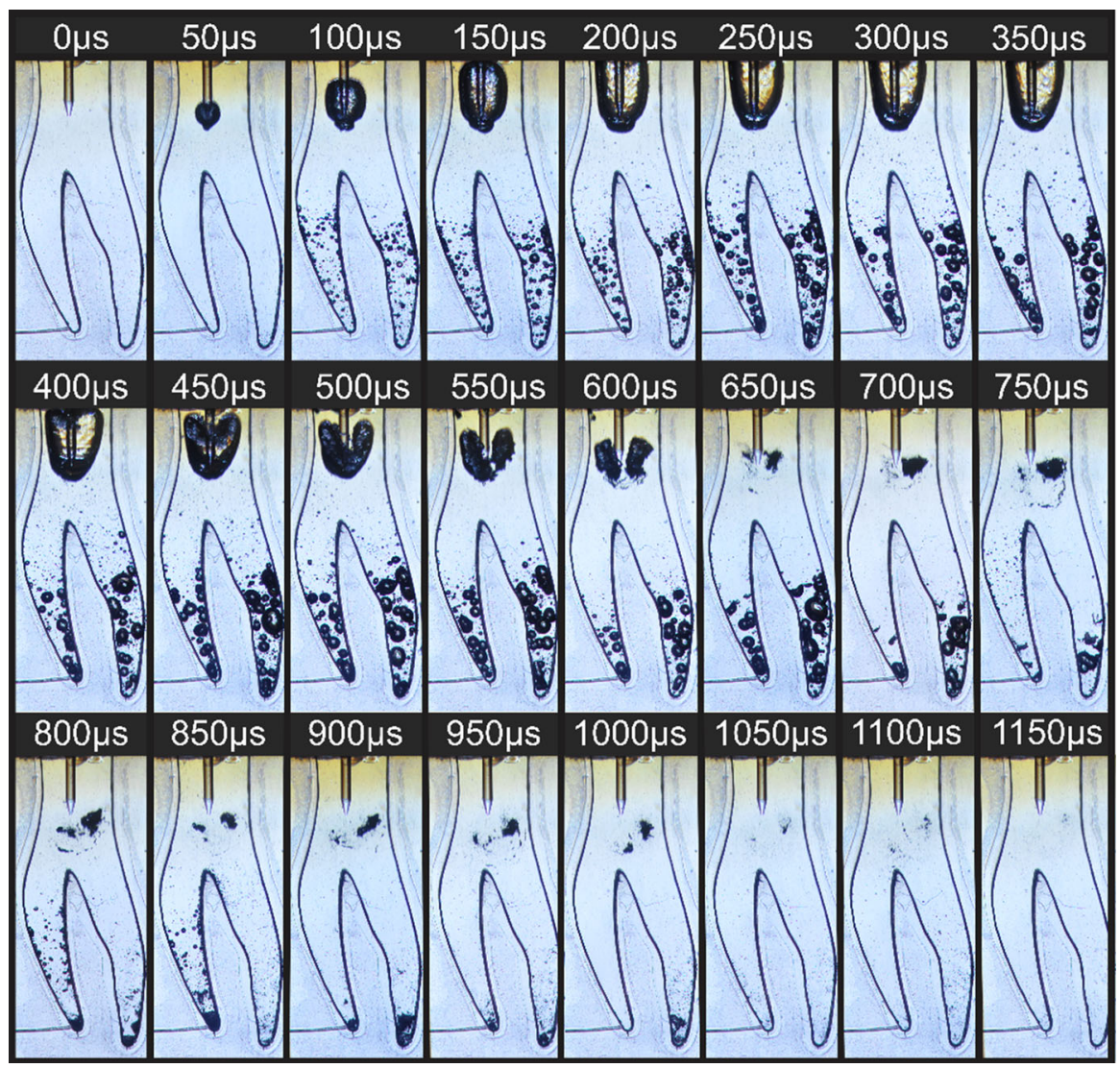

Fig. 2 The series of images shows the life cycle of a laser-induced vapor bubble. Because light of wavelength $2.94 \mu \mathrm{m}$ is strongly absorbed in water, a very thin layer of water next to the FT is heated up in a very short amount of time beyond its boiling point. This leads to explosive boiling and a rapid expansion of the cavitation bubble $(0 \mu \mathrm{s}$ to $300 \mu \mathrm{s})$. This process continues until the pressure inside the bubble is equal to the outside pressure, at which point the bubble starts collapsing $(350 \mu \mathrm{s})$. After the initial collapse of the bubble $(700 \mu \mathrm{s})$, additional oscillations can occur

\section{Experimental Results}

\subsection{Vapor Bubble Dynamics and Ultrasound Irrigation Results}

Figure 2 shows the laser-induced vapor bubble oscillation in $50 \mu$ s time intervals. In unconstrained space, laser-induced vapor bubbles have been shown to grow in a roughly spherical shape $[7,8]$. The prolate spheroidal shape of the growing bubble is likely caused by the constraints of the root canal as the water displaced by the growing bubble is forced out of the canal alongside its walls. Additionally, the bubble's oscillation inside the root canal is approximately $250 \%$ longer than in unconstrained space due to friction loss caused by increased fluid velocity between the cavitation bubble and the canal surfaces. The shock wave that typically follows the bubble's collapse $[8,12]$ is also absent. 


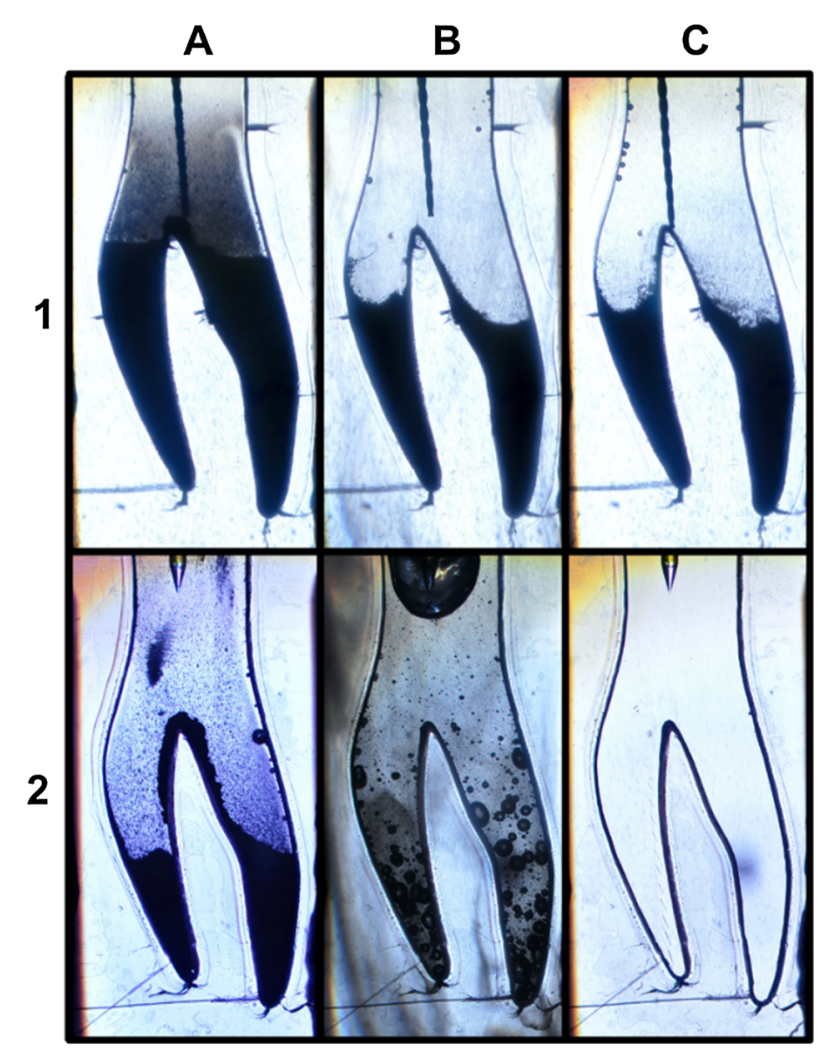

Fig. 3 A comparison between ultrasonic (row 1) and laser (row 2) irrigation. Column A shows the state of the root canal before the treatment, with the ultrasonic file/FT inserted. The laser irrigation treatment lasted for $5 \mathrm{~s}$, a total of 75 laser pulses at a frequency of $15 \mathrm{~Hz}(50 \mathrm{~mJ}$ per pulse, $3.75 \mathrm{~J}$ total energy). Ultrasound irrigation effectively ended after $2 \mathrm{~s}$ after which no further irrigation occurred even though the ultrasound device was left on for $60 \mathrm{~s}$. Column $B$ shows activity in the root canal during irrigation. The after-treatment photos (column $C$ ) were taken a minute after the end of each treatment to allow for any debris particles to settle

Figure 3 shows a comparison between ultrasound (row 1) and laser (row 2) irrigation before (column A), during (column B), and after (column C) irrigation. The ultrasonic method is effective in partially removing the simulated debris from the root canal model but its effectiveness is limited to the vicinity of the ultrasonic needle. With laser irrigation, the debris is completely removed from the root canal model. This represents a clear advantage for the laser method especially for irrigation of complex root canal geometries where portions of the root canal might not be accessible by the ultrasonic needle or a similar device.

\subsection{Water Vorticity Results}

Figure 4 shows the results of the experiment where each image represents a distinct laser pulse at various delay times. During the vapor bubble's expansion and collapse, 

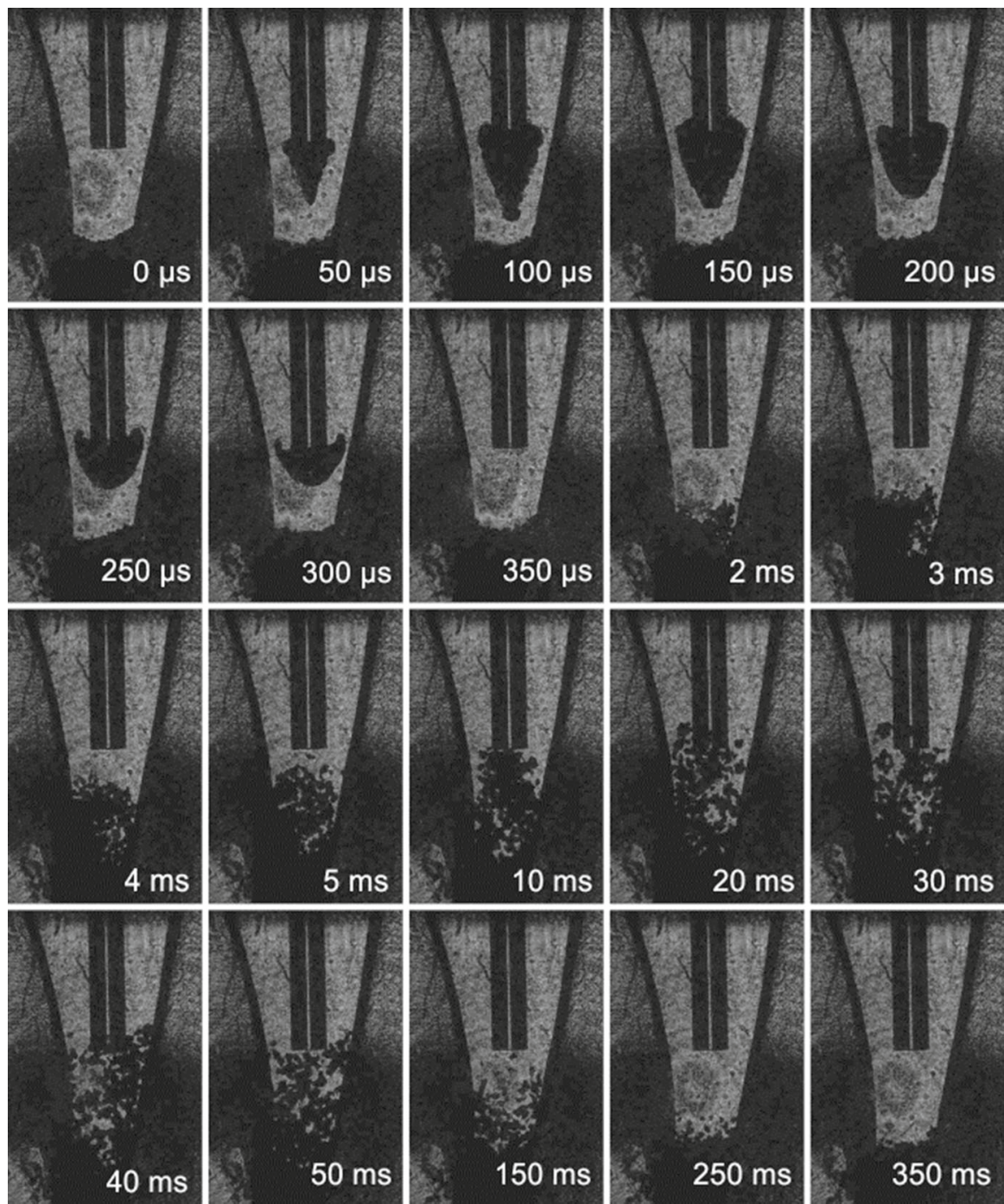

Fig. 4 The series of images shows water vorticity after a laser-induced cavitation bubble using simulated debris particles. Significant water flow can be observed $2 \mathrm{~ms}$ after the beginning of the laser pulse, which is long after the collapse of the cavitation bubble (approximately $300 \mu \mathrm{s}$ after the laser pulse). The particles settle to the ground in approximately $200 \mathrm{~ms}$ to $300 \mathrm{~ms}$

there is no observable effect on the debris. The slight variability seen in the surface of the debris sediment is due to the particles settling to the bottom of the root canal in a slightly different manner after each laser pulse. After approximately $2 \mathrm{~ms}$, water near the surface of the debris sediment becomes slightly agitated and particles start flowing upwards and mixing with the water. The speed of water flow from the fiber tip to the debris is approximately $0.75 \mathrm{~m} \cdot \mathrm{s}^{-1}$. This effect continues for $20 \mathrm{~ms}$ to $30 \mathrm{~ms}$, when the debris particles reach their maximum height, the water flow subsides, and the sediment 


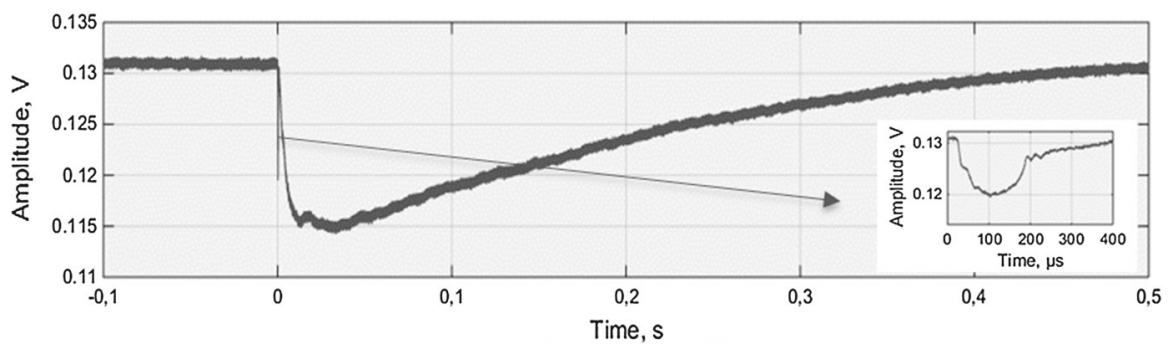

Fig. 5 Laser-beam-transmission probe (LBTP) results confirm the findings of shadow photographs in Fig. 4. LBTP signal shows the entire oscillation of a single cavitation bubble (inset) and the subsequent water vorticity of debris particles

slowly settles to the bottom of the root canal $(200 \mathrm{~ms}$ to $300 \mathrm{~ms})$. These results are confirmed by measurements with a LBTP — a typical signal is shown in Fig. 5. The negative peak at time $100 \mu$ s (see zoomed image in Fig. 5) is caused by the laserinduced vapor bubble. As the debris inside the root canal model becomes agitated and starts mixing with the water inside the canal, less light is transmitted to the photodiode and the amplitude of the signal drops. As the mixing subsides and the debris settles back to the bottom of the canal, the amplitude of the signal on the photodiode returns to the level measured before the generation of the vapor bubble. This leads us to the conclusion that water flow induced by the vapor bubble is responsible for the cleaning action inside the root canal.

\section{Conclusions}

We studied the optodynamic phenomena during ultrasonic and laser-activated irrigation within the root canal. Results confirm important phenomenological differences in the two methods with the laser method resulting in much deeper irrigation. Observations of simulated debris particles show liquid vorticity effects which in our opinion represents the major cleaning mechanism. While root canal morphologies can be very complex and variable and a single or even multiple models cannot encompass all these traits, we believe our results are a good indication of processes during laser irrigation in real root canals.

Open Access This article is distributed under the terms of the Creative Commons Attribution 4.0 International License (http://creativecommons.org/licenses/by/4.0/), which permits unrestricted use, distribution, and reproduction in any medium, provided you give appropriate credit to the original author(s) and the source, provide a link to the Creative Commons license, and indicate if changes were made.

\section{References}

1. S.D. de Groot et al., Int. Endod. J. 42(12), 1077 (2009)

2. G. Olivi, E. DiVito, J. Laser Health Acad. 1, 22 (2012)

3. E. DiVito, O.A. Peters, G. Olivi, Laser Med. Sci. 27, 273 (2012)

4. O.A. Peters, S. Bardsley, J. Fong, G. Pandher, E. DiVito, J. Endod. 37, 1008 (2011) 
5. E. Deleu et al., Lasers Med. Sci. 30, 831 (2015)

6. A. Vogel, V. Venugopalan, Chem. Rev. 103, 577 (2003)

7. P. Gregorčič et al., J. Biomed. Opt. 17, 075006 (2012)

8. M. Malki et al., J. Endod. 38, 657 (2012)

9. L.W. van der Sluis et al., Int. Endod. J. 40, 415 (2007)

10. P. Gregorčič et al., J. Laser Health Acad. 2014, 14 (2014)

11. W. Lauterborn, T. Kurz, Rep. Prog. Phys. 73, 106501 (2010)

12. R. Petkovšek, P. Gregorčič, J. Appl. Phys. 102, 044909 (2007) 